\title{
Lenguaje inclusivo y representación del colectivo LGTBIQ+ en solicitudes de adopción: análisis contrastivo inglés-español
}

\section{Inclusive language and queer representation in adoption applications: a contrastive English-Spanish analysis}

\author{
IRENE RODRíGuez ARCos \\ irene.rodriguez@uneatlantico.es \\ Universidad Europea del Atlántico \\ ANDREA GonZÁLEZ DíAZ \\ andrea.gonzalez@alumnos.uneatlantico.es \\ Universidad Europea del Atlántico
}

Fecha de recepción: 10 de septiembre de 2020

Fecha de aceptación: 16 de febrero de 2021

Resumen: El lenguaje inclusivo constituye una forma simbólica de definir pertenencia a una comunidad o un intento de reflejar identidades y orientaciones que van más allá de la visibilidad de la mujer. Aunque se abren camino diferentes estrategias para lograr una mayor representación de ciertos colectivos, no todas resultan igual de aceptables en lo que se refiere a la esfera administrativo-legal. Justificando la posibilidad teórica de desestabilizar el género y la responsabilidad ética de reconocer todo el espectro que abarcan las siglas LGTBIQ+, se estudia una muestra compuesta por documentos de adopción en español y en inglés, que permitirá observar hasta qué punto se logra contemplar la presencia de una comunidad que es susceptible de tener que recurrir a estos instrumentos legales. Además de comprobar la efectividad de las estrategias utilizadas en ambas lenguas con propuestas fundamentadas desde el plano de la Lingüística, como la de Grijelmo, se reflexionará acerca de qué fórmulas resultan aceptables en este contexto específico de uso, en el que, si bien es cierto que se han producido algunos avances, aún queda camino por recorrer. No obstante, ambas lenguas disponen de mecanismos propios del lenguaje administrativo-legal que no obligan a incorporar neologismos ni dobletes y están perfectamente asentadas socialmente, por lo que se defiende la necesidad de estudiarlas e incluirlas para lograr un reconocimiento que el colectivo aún lucha por conseguir.

Palabras clave: Lenguaje inclusivo, Género, LGTBIQ+, Hospitalidad, Performatividad. 
Abstract: Inclusive language represents a symbolic way of defining the belonging to a community or an attempt to reflect identities and orientations beyond the visibility of women. Although some strategies are starting to be used in order to achieve a more accurate representation of some groups, not all of them are totally acceptable in administrative and legal fields. By justifying the theoretical possibility of destabilizing gender and the ethical responsibility of acknowledging the whole spectrum included in the LGBTIQ+ denomination, this paper studies a corpus of adoption applications in Spanish and English, which allows to explore to which extent the LGBTIQ+ community is represented, a social group who may experience the adoption process. Validating the effect of the particular use of inclusive language in both languages, resorting to studies from Spanish Linguistics such as Grijelmo's, the paper will also analyse formulas which may be acceptable in this specific context of use, where some advances have been made, but deeper research is needed. However, both languages have their own mechanisms, belonging to the administrative-legal context of use, which do not force speakers to include neologisms or artificial formulas, and are socially accepted: this is why there is a need to study and use them to achieve a representation which the LGBTIQ+ members are still fighting to see.

Keywords: Inclusive language, Gender, LGBTIQ+, Hospitality, Performativity.

\section{INTRODUCCIÓN}

El movimiento feminista y las reivindicaciones del colectivo LGTBIQ+ se han ido abriendo hueco en los espacios públicos. Desde que un histórico 8 de marzo de 2018 las marchas feministas tuvieran presencia global, el debate y la lucha por una igualdad real de derechos básicos han ocupado páginas de la prensa generalista y se han convertido en objetos de estudio innovadores e interdisciplinares desde la academia (Sánchez y Fernández, 2019). Una piedra angular de ambos movimientos ha sido el reconocimiento social y, con él, la obtención de una representación de identidades de género y sexuales más amplia y acorde con la realidad social a la que asistimos.

Una de las herramientas que pueden vehicular estas representaciones es lo que ha venido llamándose «lenguaje inclusivo», un tema tan candente como controvertido, que ha sido celebrado y denostado a partes iguales. Finalmente, se empieza a contar con obras firmadas por autores de prestigio (Grijelmo, 2019) que analizan y estudian el fenómeno más allá de los dobletes y el uso de otros signos que caen fuera de lo normativo, argumentos de los que se han servido los detractores de su uso en diferentes ocasiones para desprestigiarlo. Por lo tanto, uno de los objetivos del trabajo consistirá en profundizar en las estrategias existentes en español (por contraposición al 
inglés, que ofrece más facilidades que otras lenguas al contener menos - $u$ otro tipo de - marcas de género) para lograr una mayor inclusión. Y decimos mayor, porque sí se han hecho intentos de abarcar las diferentes sexualidades e identidades, pero nuestra hipótesis es que, probablemente, no los suficientes y desde un enfoque que merece ser revisado académicamente. Para demostrarlo, se ha confeccionado un corpus con solicitudes de adopción en inglés y español y se ha analizado la presencia del lenguaje inclusivo en ambas lenguas. Las razones por las que se ha escogido este género textual es que, aunque en situaciones informales y en la academia se puedan hacer intentos de transformar la lengua, donde realmente se palpa si se han producido cambios es en las esferas administrativas y legales, entornos en los que la lengua se ha seleccionado cuidadosamente. Por esto, y porque este tipo de documentación es susceptible de ser utilizada en alguna ocasión por el colectivo LGTBIQ+, se ha querido estudiar en qué medida la lengua representa su identidad y su lucha o, en otras palabras, cuánto retraso se observa a la hora de reflejar unos avances sociales que, por suerte, ya son el presente (desde que se legalizara el matrimonio homosexual en España hace ya quince años o la Proposición de Ley 122/000191 sobre la protección jurídica de las personas trans y el derecho a la libre determinación de la identidad sexual y expresión de género).

En palabras de Grijelmo (2019, p. 52), aunque la lengua no cambia la realidad, «puede servir para que la realidad cambie. $Y$ también tiene la capacidad de disfrazarla, esconderla». Si bien es cierto que ya se han dado los primeros pasos a nivel social, resulta relevante y revelador profundizar en cómo nos construyen las instituciones a nivel lingüístico, empezando por nuestra identidad y el propio concepto de «género».

\section{EL GÉNERO Y LA IDENTIDAD COMO CONSTRUCTOS SOCIALES Y LINGÜÍSTICOS}

Antes de continuar, es relevante explicar el trasfondo de las siglas que se usan a lo largo de la investigación. El debate sobre cuáles son las más apropiadas para denominar a la comunidad no está exento de análisis previo. Desde sus orígenes, este debate ha estado muy presente, remontándose a la inclusión de la «T» y la «B» en la comunidad, que anteriormente estaba reservada a la homosexualidad. No todo el mundo aceptó en la misma medida la inclusión de personas trans y bisexuales, que incluso llegaron a sufrir rechazo dentro de una comunidad que se había formado para la aceptación (Alexander, 2003). Los horizontes de esta comunidad son cada vez más amplios, y ya no es difícil encontrar siglas que añadan a las personas intersexuales, observar queer usado como término genérico, el reconocimiento de asexuales, etc., una amplitud que en ocasiones se resuelve mediante la inclusión de un símbolo del más $(+)$ al final de las siglas.

Hikma 20 (1) (2021), 157 - 184 
Estos avances pueden parecer desmesurados, pero si recordamos que, en su momento, la «T» y la «B» también fueron rechazadas, es sencillo comprender por qué tanta gente no considera excesiva la presencia de estas letras. Finalmente, tras comparar varios documentos, el trabajo se decanta por el uso de las siglas LGTBIQ+, ya que se han observado en la academia (Sánchez y Fernández, 2019; fundación pionera en el mundo de los estudios queer en España). Esta reflexión refleja, por un lado, la necesidad de ampliar el alcance de la comunidad para acoger personas que, de una forma u otra, se alejan de la norma establecida, y, por otro, la utilidad de usar las mismas siglas en todas las investigaciones académicas relacionadas con este tema en términos de coherencia y consistencia terminológica.

\subsection{Desaprender el género}

Ciertas corrientes feministas (Butler, 1990/1999) han insistido desde hace décadas en «desaprender» el género como algo estable, una parte inmutable e inevitable de nuestra identidad social, y rechazan lo establecido en lo que concierne a la sexualidad e identidad. Las bases de las teorías queer y feministas se han criticado mutuamente y se han motivado para crear y actualizar sus fundamentos a través de los años y de los contextos socioculturales, tratando de recoger e incluir a todas y cada una de las piezas que forman una sociedad, sin olvidar a las personas racializadas, neurodivergentes o con diferentes capacidades físicas, en el intento de lograr un mayor grado de inclusión. Este corte postestructuralista, que articula nuestra investigación, nos será de utilidad posteriormente para incorporar conceptos derrideanos como el de «hospitalidad» (Derrida, Hostipitality, 2000).

Estas teorías narran cómo ya se tenía presente la necesidad de una modificación lingüística, y señalaban que «the development of a language that fully or adequately represents women has seemed necessary to foster the political visibility of women" (Butler, 1990/1999, p. 22). Es innegable que las mujeres de la época ya eran conscientes de su situación sociocultural y sentían que su representación era insuficiente, o incluso en ciertos ámbitos, inexistente. Pero en esta ocasión el estudio va más allá, tratando de desmontar las ideas preconcebidas que se puedan tener del propio género, que se ve afectado por una multitud de factores socioculturales. En este sentido, resulta casi inevitable volver a Simone de Beauvoir, con una de sus frases más famosas: «no se nace mujer: se llega a serlo» (Beauvoir, 1949/2016, p. 87). Butler, muy influida por las ideas feministas de Beauvoir, usa la base de estas teorías para hacer florecer muchas cuestiones relacionadas con la propia naturaleza del género. $\mathrm{Si}$ las propias características del género son tan inestables, volátiles y variables, ¿es nuestro género una elección puramente voluntaria? Beauvoir ya nos indicaba 
que, para ella, esta elección se debe, en esencia, a los condicionantes y presiones externas, a la cultura en la que cada individuo nace y crece. Esto queda muy alejado de los condicionantes físicos que, según se ha considerado socialmente, van estrechamente enlazados con el "sexo» de una persona (Beauvoir, 1949/2016). Dicho en otras palabras, Beauvoir se aleja de la idea de que ciertas características biológicas y genitales marquen inconfundiblemente la idea de género inherente. Para esta autora, el cuerpo no es más que una situación, y lo que se conocía por "sexo», suele ser una referencia mucho más fuerte a los condicionantes sociales del género, más que a los condicionantes corporales.

Butler (1990/1999) se centra en el concepto de «performatividad», que define como el refuerzo, muchas veces inconsciente, que realizan los individuos al tratar de seguir las normas de su género predeterminado, estipulado por sus condicionantes biológicos. La identidad que estas personas expresan socialmente se preserva gracias a sus acciones, pero esa identidad carece de una base tangible, escrita. Dicho en otras palabras, es un bucle en el que a un individuo con unas ciertas condiciones físicas se le fuerza involuntariamente a cumplir unos modelos de comportamiento, y mediante la performatividad de esas características, está reforzando los estereotipos de su género; por ende, hace cada vez más difícil el salirse de estos márgenes de forma involuntaria. La pregunta ahora reside en por qué los individuos sienten tanta presión por ajustarse a estos modelos, si son en realidad tan frágiles, volátiles, y están tan subyugados a tantísimos aspectos externos.

Los propios individuos van formando la idea de género de su situación, en muchas ocasiones alejado de la matriz heterosexual. La disputa entre lo que siempre se ha concebido como género y sexo no es algo novedoso, únicamente se ha empezado a documentar y visibilizar con más cotidianidad en los últimos años, como se puede ver en autores como Martínez Pleguezuelos (2018). La «falta de continuidad» de estas personas, que se recogen bajo las identidades trans y no binarias (en contraposición a la norma cisgénero) no es más que un rechazo hacia la identidad establecida, socialmente instaurada. La mera presencia de estas personas con género «incoherente» rompe la aparente firmeza de este muro, estos moldes a los que les han enseñado que se deben ceñir. Debido a los avances sociales y a la lenta, pero presente representación de estas personas que se alejan de las identidades cis, ya son una pequeña realidad en los contextos de casi todas y todos. Las «faltas» en este muro binario empiezan a ser más y más evidentes, y aunque siguen viéndose como un problema a los ojos de muchos individuos, empiezan a ser celebradas por ciertos colectivos. El problema surge cuando aquellas personas son conscientes de su situación 
sociocultural, y sienten que su representación es defectuosa, incluso en ciertos ámbitos, inexistente (Martínez Pleguezuelos, 2018).

\subsection{La matriz heterosexual y la regulación de los cuerpos}

Hasta aquí se ha expuesto la reificación involuntaria de las ideas de género, de identidad, de lo que somos, en contraposición a lo que se nos enseña a ser. Pero ahora el foco de atención se dirige al otro gran pilar de la comunidad LGTBIQ+; el interés romántico, afectivo o sexual, o incluso la falta de los mismos. Para referirse a este aspecto, Butler referencia a Foucault, con quien comparte su visión posestructuralista:

For Foucault, the substantive grammar of sex imposes an artificial binary relation between the sexes, as well as an artificial internal coherence within each term of that binary. The binary regulation of sexuality suppresses the subversive multiplicity of a sexuality that disrupts heterosexual, reproductive, and medicojuridical hegemonies (Butler, 1990/1999, pp. 25-26).

Entender la normativa de atracción sexual entre «hombres» y «mujeres» es imposible si no entendemos los orígenes de esta diferenciación entre «hombres» y «mujeres». El deseo heterosexual entre personas consideradas cis se basa en una relación de oposición entre el género de un individuo y "su contrario". La heterosexualidad se ha coronado como algo institucional, el producto del sistema de géneros binario y opuesto. Si seguimos centrándonos en las ideas que Butler comparte con Foucault, veremos que la autora señala que establecer un régimen relativo a la sexualidad, una herramienta que regule la experiencia sexual y determine las orientaciones es, al igual que los aspectos de género vistos anteriormente, una respuesta a los márgenes establecidos. Foucault ya daba voz en su libro Historia de la sexualidad: la voluntad del saber a las personas lesbianas y gays, compartiendo que la homosexualidad se puso a hablar de sí misma, a reivindicar su legitimidad o su «naturalidad» incorporando frecuentemente al vocabulario las categorías con que era médicamente descalificada (Foucault, 1976/2007). Igualmente, en Vigilar y castigar (1975/2002), el autor alertaba de cómo ciertos discursos autoritarios o "disciplinas» creaban "cuerpos dóciles», que actúan "como se quiere, con las técnicas, según la rapidez y la eficacia que se determina. La disciplina fabrica así cuerpos sometidos» (Foucault, 1975/2002, pp. 141-142). Indudablemente, la medicalización de ciertos sujetos, como forma de adaptarlos y traducirlos a los sistemas de lo familiar, de la voz autoritaria, supone una violencia innegable, ante la que éticamente deberíamos estar comprometidos (Braidotti, 2006/2009) a adoptar una actitud de hospitalidad (Derrida, 2000). Una de las vías para su puesta en práctica podría ser la de dotar a estos sujetos de reconocimiento legal y 
político; es decir, de existencia en el espacio público, en el que puedan tener voz y que se les reconozca su discurso (Beard, 2017).

\subsection{Lengua, identidad y traducción: la necesidad de hospitalidad lingüística}

El vínculo entre lengua e identidad es indisoluble tal y como señala Butler en Cuerpos que importan (1995/2002): «las palabras por sí solas tenían el poder de modelar los cuerpos en virtud de su propia sustancia lingüística» (Butler, 1995/2002, p. 12). Y en este sentido, la relación con la Traductología es innegable: Cronin, en su obra Translation and Identity (2006), defiende el trasvase que implica el acto de traducir. Además de un intercambio lingüístico, supone destacar las diferencias que caracterizan a cada individuo. Igualmente considera que las distintas identidades no deben limitarse a un único contexto, y que la traducción puede abrir las puertas internacionales para que estas identidades se expandan y visibilicen, creando así una vereda para el diálogo y la eliminación de las represiones basadas en la identidad (Cronin, 2006). Asimismo, las estrategias de traducción que contribuyen a la invisibilización de ciertos colectivos conllevan consecuencias de índole ética de las que la persona que ha desempeñado la traducción es responsable (Baker, 2006). contrario:

La cuestión es que la identidad se asume hasta que se demuestra lo

[M]ost components of identity become noticeable only when they are activated by specific circumstances. And one of these special circumstances is translation and other forms of intercultural communication. They trigger and awareness of cultural identities (House, Martín y Baumgarten, 2005, p. 4).

Y cuando el poder lo hace visible, empieza el conflicto, la desigualdad y la discriminación. Derrida (2000), desde su visión postestructuralista, ya alertaba de cómo ciertas corrientes hegemónicas siempre han determinado la inclusión de ciertos colectivos en sus sistemas de poder. Sin embargo, basándose en la ética kantiana, nos recuerda que, por el mero hecho de ser humanos, debemos al Otro cierta forma de «hospitalidad», pues «ethics is hospitality» (Derrida, 1977/2001, p. 17). Como bien señala el filósofo, la hospitalidad no puede darse sin cierto comoponente de hostilidad (Derrida, 2000), en el que el patrón debe salir al encuentro de su huésped y abandonar su zona de lo familiar. Este encuentro es necesario para que se produzca la inclusión, porque si el Extraño, en este caso las personas que caen fuera de la matriz heterosexual en términos de identidad de género u orientación sexual, realmente estuvieran en igualdad de derechos y accesibilidad, no estaría produciéndose este debate: 
$[\mathrm{H}]$ ospitality opens spaces up as a form of exchange; it dialogues with space and border theory, reexamines the roles of hosts and guests, mobilizes the notion of home and converses with hospitable and inhospitable languages as it rearranges the concepts of belonging, membership and citizenship (Manzanas y Benito, 2017, p. 8).

Entendemos, con autoras como Vidal (2014, p. 245, 256), que el lenguaje constituye un locus en el que surgen asimetrías de poder y cultura, por lo que no resulta extraño que Manzanas y Benito (2017) hablen de él como un aspecto crucial de la hospitalidad, algo de lo que Ricoeur ya era consciente al acuñar el concepto de «hospitalidad lingüística» (Ricoeur, 2005), sin el cual no seríamos sensibles a la extranjeridad de la propia lengua. Reflexionar acerca de los límites y las carencias de lo conocido no solo es responsable, sino necesario. Y aunque, como advierte Grijelmo (2019), en el lenguaje oral es casi imposible poner en práctica ciertas recomendaciones lingüísticas, el lenguaje inclusivo es algo que debe estar presente en la «lengua cultivada»:

[S]e pueden modificar las lenguas naturales con diversas finalidades [...]. Estas lenguas han de ser aprendidas y enseñadas mediante instrucción específica por parte de los que las dominan y no pueden ser adquiridas de modo natural por los infantes como ocurre en el caso de las lenguas naturales. [...] se usa habitualmente en público y requiere de un esfuerzo a cargo de quien habla o escribe (Grijelmo, 2019, p. 34).

De esta manera, en contextos administrativos o legales, en los que la lengua que se emplea se encuentra mediada de forma significativa, planteamos la necesidad y la obligación de que esto mismo se haga para lograr una mayor inclusión de las personas que pertenecen al colectivo LGTBIQ+. Aunque requiera un mayor esfuerzo, ya se han expuesto las razones éticas que respaldan el llevarlo a cabo. En el siguiente epígrafe se plantearán posibles formas de hacerlo, sin caer en construcciones artificiales o morfemas novedosos que no se han asentado aún en la lengua.

\section{EL LENGUAJE INCLUSIVO: ESTRATEGIAS EN ESPAÑOL Y EN INGLÉS}

Grijelmo (2019) expone con claridad y rigor los diferentes mecanismos de la lengua española para comunicar con generalidad: el controvertido masculino genérico que, para ciertas corrientes, supone una sobrerrepresentación masculina o androcéntrica (Franco, 2019) o los manidos dobletes. Sin embargo, el lingüista también señala el matiz de diferencia entre el masculino genérico (inclusivo) y masculino sexuado (Grijelmo, 2019, p.14), pues no ha de olvidarse que el femenino aparece en las lenguas ante la necesidad de darle visibilidad a un colectivo que se había 
significado previamente como neutro, y no necesariamente masculino. Tras un recorrido en el que analiza el uso de dobletes, incluyendo una hipotética modificación de la Constitución Española con ciertas fórmulas redundantes (y sin validez jurídica real, tan solo simbólica), o las connotaciones de expresiones como «violencia de género» (Grijelmo, 2019, pp. 151-160), el autor llega a una propuesta que él mismo califica de «borrador», para ser discutida y mejorada, que incluye recomendaciones para la ya citada «lengua cultivada».

Estas estrategias abarcan, por ejemplo, el uso de sustantivos colectivos para evitar otros términos que contengan marcas de género (alumnado, profesorado). Asimismo, el lingüista recuerda las Máximas de Grice acerca de la comunicación (principios de cooperación y relevancia), critica el uso de refranes y chascarrillos populares que ofrecen una representación sesgada de la mujer y otros colectivos y señala que el lenguaje inclusivo es un lenguaje «identitario», que muestra preocupación por las desigualdades sociales y revela ciertas posiciones ideológicas. No obstante, el no usarlo de manera sistemática (especialmente en lengua hablada y situaciones coloquiales, pues equivaldría a aprender un nuevo idioma), no debería ser motivo de crítica o insulto hacia los hablantes (Grijelmo, 2019). De igual manera, se desecha el uso de caracteres especiales como la @ o la $x$, que presentan especiales dificultades en textos susceptibles de ser oralizados. En el caso concreto de los documentos analizados, pertenecientes a la esfera administrativa, se ha comprobado que, además de presentar inconsistencias lingüísticas y terminológicas a la hora de incluir dobletes, también existen fórmulas perfectamente asentadas en el lenguaje jurídico-administrativo del español que podrían funcionar sin deformar la lengua: por ejemplo, podría sustituirse un «Deseo ser notificado/a» por «Deseo que se me notifique».

En lo que respecta a la lengua inglesa, las estrategias son parecidas. Desde hace décadas se cuenta con obras que recogen propuestas alternativas (Miller y Casey, 1995), desde romper con el androcentrismo o el problema de los pronombres. Si bien es cierto que en inglés hay menor presencia de marcas de género, existen sustantivos marcados como «father» 0 «mother» que pueden ser sustituidos por «parent». También se puede recurrir al uso de dobletes que, como en el caso anterior, son más escasos. Por último, parece que se está asentado el uso de «they» $y$ «them» como pronombres inclusivos, algo que en español está surgiendo con la forma «elle» (Maldonado, 2017), pero el uso no está muy extendido fuera de ciertos colectivos. En este último caso, la novedad es trascendental para ciertas personas que no se sienten representadas ni con el masculino ni con el femenino, de género no binario. En todos estos casos, convenimos con 
Grijelmo (2019) en que primero se tiene que producir un cambio social, y con el tiempo, las lenguas, moldeadas por los hablantes y transigentes con ellos, desarrollarán sus propias estrategias, incorporando neologismos o transformándose, hasta el punto de que ciertas marcas queden como algo testimonial (ibid.: p. 283) en la comunicación.

\section{Metodología Y Descripción De La Muestra}

Para llevar a cabo esta investigación se han analizado un total de veinticinco documentos relacionados con la adopción, seis en español y diecinueve en inglés. La disonancia al comparar estas cifras responde a la diferencia de comunidades autónomas en España y estados en Estados Unidos, por lo que ambas muestras son representativas. Todos los archivos analizados se han recuperado de las páginas web de los veinticinco gobiernos correspondientes, por lo que se consideran vigentes y fiables. Se han analizado todos los documentos que ofrecía cada país de forma sencilla, virtual y gratuita. Estas diferencias en cuanto a la accesibilidad responden a las diferencias legales de cada zona, además de las restricciones propias de la situación de pandemia global que sucedía en el momento de redactar este artículo. Por ejemplo, en casos como el de Andalucía, se requiere una entrevista previa para acceder al trámite, por lo que no fue posible la obtención de los documentos de forma inmediata.

El porqué de esta temática documental se basa en que pertenecen a lo que en el marco teórico hemos presentado como «lengua cultivada». A nivel cotidiano, y diario, la lengua es mucho más moldeable y tangible. Estas fluctuaciones en el uso particular de los hablantes suponen el primer paso para un potencial cambio en la lengua a niveles administrativos, políticos, o legales, ámbitos donde el lenguaje es mucho más tradicionalista y reacio a sufrir cambios. La lengua es un elemento vivo y en constante metamorfosis, pero la velocidad con la que se incorporan estas variaciones es muy diferente dependiendo del ámbito en el que centremos la investigación.

Para realizar esta tarea y ser conscientes de la posible discriminación que sufren estas personas, es necesario recopilar un corpus que dé muestras de su reconocimiento y visibilidad a nivel administrativo-legal. De esta manera, se han obtenido documentos internacionales y vigentes sobre los procesos de adopción, un tipo de archivo con un enorme potencial de acabar en manos de una unidad familiar LGBTIQ+. Una vez recuperados los documentos, se realiza un análisis de los mismos separándolos por idioma, ya que las características y técnicas de los diferentes idiomas para lograr esta inclusión puede variar. En español se analizan dobletes, términos genéricos, fórmulas administrativas y caracteres carentes de marcas de género, y en inglés se analizan dobletes, términos genéricos, fórmulas administrativas y el 
uso del neutro «they». Una vez analizado el lenguaje, el foco de atención pasa a la obligación de indicar el género o sexo (dependiendo de la elección del documento original). La forma de solicitar el género y la obligación de elegir entre dos sexos también debe ser objeto de estudio, ya que puede indicar una falta de reconocimiento hacia las identidades disidentes, en este caso el género no binario. Con toda esta información se puede obtener una conclusión que indique las tendencias de estos textos, con sus correspondientes diferencias entre los dos idiomas, y la (posible falta de) representación que se ha logrado obtener.

La muestra en español está compuesta por documentos relacionados con la adopción nacional de seis comunidades autónomas; Aragón, Cataluña, Madrid, Mallorca, Murcia y La Rioja. La elección de estos documentos se debe a la legalidad de cada comunidad, que causa variaciones en cuanto a la privacidad y accesibilidad de estos archivos. Los documentos analizados fueron facilitados por las páginas de los gobiernos de cada comunidad en el momento en el que se realizó este trabajo. Las zonas no representadas responden a las diferencias legales y burocráticas, ya que en muchas zonas del país se requiere una entrevista personal para considerar las aptitudes de la familia interesada y posteriormente dar acceso a estos documentos de adopción. Esto hace que la muestra sea representativa en cuanto al panorama en España, ya que se analizan todos los archivos de acceso oficial, público y gratuito. Todos los documentos analizados se han descargado de las páginas de los gobiernos de cada comunidad autónoma, por lo que se consideran documentos recientes y vigentes independientemente de las variaciones que puedan representar en cuanto a sus últimas fechas de revisión.

Para el caso del inglés, el análisis se centra en diecinueve documentos de adopción pertenecientes a Estados Unidos, en este caso se representan los estados de Alaska, Arizona, Arkansas, California, Colorado, Delaware, Florida, Indiana, Massachusetts, Michigan, Nevada, Nueva Jersey, Nueva York, Nuevo Hampshire, Nuevo México, Tennessee, Texas, Utah y Virginia Occidental. Una vez más, su elección se debe a las particularidades legales de cada estado y a la accesibilidad a través de las páginas oficiales de cada gobierno, además de que luego se dan variaciones dependiendo del tipo de adopción del que se trate (menores en situación de orfandad, adopción internacional, etc.), por lo que se ha configurado una muestra que pudiera ser comparable a nivel de macroestructura y contenido. Esto genera una muestra representativa ya que se analizan todos los archivos de acceso oficial, público y gratuito, y de nuevo, se consideran documentos recientes y vigentes independientemente de las variaciones que puedan representar en cuanto a sus últimas fechas de revisión. 


\section{ANÁLISIS DE FÓRMULAS INCLUSIVAS EN ESPAÑOL}

\subsection{Dobletes}

Esta técnica tan popular para crear inclusión de género consiste en incluir una «a» 0 «as» acompañada de una barra o unos paréntesis junto a la palabra con género gramatical masculino. Además de ser una herramienta útil para aumentar la inclusión de género, también se usa para crear variaciones de número. Un ejemplo sacado del documento aragonés puede ser «hijos/as biológicos/as o adoptados/as». Esta opción tiende a ser problemática, ya que es fácil olvidarse de añadir todos los dobletes necesarios para una correcta concordancia y no es difícil encontrar documentos en los que se pasa por alto usar asimismo el doblete en el artículo, como podría ser «los hijos/as», que podría dar a entender que «los hijas» es una opción aceptable, como señala Grijelmo (2019). Además, el uso excesivo de dobletes puede resultar cargante para nuestro público, siendo «artificiosos e innecesarios desde el punto de vista lingüístico» (RAE, 2020), por lo que se recomienda su uso en la justa medida y cuando la opción anterior no es viable. Además, otro punto en contra es que pueden crear una brecha aún mayor entre lo considerado masculino y femenino (Horno, 2018).

Cuatro de los seis documentos españoles presentan alguna forma de doblete, por lo que es una técnica razonablemente popular. Estos dobletes muestran un esfuerzo activo por lograr esta inclusión lingüística (Grijelmo, 2019), pero, insistimos, no es sencillo mantener la concordancia durante todo el texto. Es por este motivo que en los cuatro documentos que presentan dobletes se da algún tipo de falta de continuidad, ya que no se mantiene la misma norma al utilizar esta técnica. En estas tablas se recogen algunos ejemplos extraídos de los archivos que escenifican la complejidad de mantenerse constante al utilizar esta técnica.

\begin{tabular}{|l|l|}
\hline \multicolumn{2}{|c|}{ Ejemplos de dobletes extraídos del documento de La Rioja } \\
\hline Domiciliado/a/s en & Dobletes de género y número \\
\hline No han sido declarado/s no idóneos & $\begin{array}{l}\text { Dobletes de número, a excepción de } \\
\text { la palabra «idóneos», que se } \\
\text { mantiene con plural }\end{array}$ \\
\hline $\begin{array}{l}\text { Deseo ser notificado/a de forma } \\
\text { electrónica }\end{array}$ & Dobletes de género \\
\hline $\begin{array}{l}\text { Deseo ser notificado mediante correo } \\
\text { postal }\end{array}$ & Sin dobletes \\
\hline
\end{tabular}

Tabla 1: Dobletes de La Rioja

Fuente: Gobierno de La Rioja, Políticas Sociales, Familia, Igualdad y Justicia (2016) 


\begin{tabular}{|l|l|}
\hline \multicolumn{2}{|c|}{ Ejemplos de dobletes extraídos del documento de Madrid } \\
\hline EVllos solicitante/s declara/n: & Dobletes de número para \\
Que D./Dña [...] y D.JDña [...] desean & «solicitantes» \\
ofrecerse para recibir a un menor en & Dobletes de género para «D./Dña» \\
adopción & Sin dobletes para «un menor» \\
\hline
\end{tabular}

Tabla 2: Dobletes de Madrid

Fuente: Comunidad de Madrid, Asuntos Sociales (2015)

Ambos casos muestran una clara falta de continuidad en un mismo documento, incluso en un mismo párrafo o apartado, ya que las variaciones de género y número en nuestro idioma provocan una necesidad constante de marcar desdobles si se desea mantener esta globalidad a la hora de representar las diferentes opciones contempladas. Un uso discontinuo de estos dobletes puede sugerir incongruencias gramaticales; si recuperamos la segunda tabla y nos ponemos en situación de que una pareja de mujeres llevase a cabo la adopción de dos niñas gemelas (ya que en el documento se contempla la adopción de menores pertenecientes a la misma familia), el documento sugeriría la frase «Los solicitantes declaran: Que Dña [...] y Dña [...] desean ofrecerse para recibir a un menor en adopción». En ese caso, el léxico no lograría representar la situación real, a pesar de la cantidad de dobletes que se han utilizado. Es una técnica que puede resultar sencilla y ventajosa, pero muy poco favorable para el texto si no se mantiene de forma regular.

\subsection{Términos genéricos}

Esta técnica, muy eficaz a la hora de crear representación, consiste en eliminar las palabras que contengan una fuerte carga de género por otras más generales. Para lograrlo, se hace que sea una nominalización general la que lleve la marca de género, y no la palabra que representa a las personas, usando perífrasis como «el grupo de», «el personal», «las personas que», etc. Por otro lado, el uso de las palabras sin marca de género que ofrece nuestra lengua nos puede facilitar también la tarea. Siguiendo con la temática adoptiva que contemple familias muy diversas, se puede sustituir "padre adoptivo» $y$ «madre adoptiva» por «solicitante A» $y$ «solicitante B». Esta es una herramienta con la que logramos, de forma sencilla, un lenguaje más inclusivo, pero una vez más, los artículos en español nos pueden entorpecer de manera casi inconsciente, de forma similar al caso de los dobletes. Esto quiere decir que, si la expresión usada es «los solicitantes», a pesar de que el término «solicitantes» contemple a dos mujeres o dos personas de género no binario, el artículo sugiere un masculino genérico. 
El uso de estos términos genéricos está presente en todos los documentos analizados, en el que destacan los términos «solicitante» y «menor» cuando se habla de la parte adoptante y adoptada, respectivamente. En tan solo uno de los documentos, el de Mallorca, aparece el término "padres», aunque cabe destacar que se usa como referente para la familia de quienes están llevando a cabo la adopción. Los documentos denotan en su mayoría un nivel más elevado de inclusión al referirse a la parte adoptante, es decir, a quien rellena el propio formulario (aunque esto no exime de faltas a los documentos, como el ya expuesto caso de usar «el solicitante» con un artículo masculino). El trato hacia la parte adoptada es mucho más inestable, y se tiende a decantar por el masculino genérico «hijos», a excepción de momentos puntuales en los que sí se usa el término genérico «menor». En el caso de otros núcleos familiares, la inclusión disminuye, tal y como se ha visto en el ejemplo de Mallorca y su masculino genérico «padres». A continuación se incluyen ejemplos de diferentes textos, que consiguen en mayor o menor grado lograr esta representación:

\begin{tabular}{|l|l|}
\hline \multicolumn{2}{|c|}{ Ejemplos de téminos genéricos extraídos del documento de Cataluña } \\
\hline Datos de identificación del solicitante & $\begin{array}{l}\text { Témino genérico «solicitante», que pierde la } \\
\text { inclusividad al estar acompañado del } \\
\text { masculino «del» }\end{array}$ \\
\hline $\begin{array}{l}\text { Datos de identificación de la persona } \\
\text { solicitante }\end{array}$ & $\begin{array}{l}\text { Término genérico «solicitante», que mantiene } \\
\text { la inclusividad al estar acompañado del } \\
\text { término genérico «persona» }\end{array}$ \\
\hline
\end{tabular}

Tabla 3: Términos genéricos de Cataluña

Fuente: Generalitat de Catalunya (2020)

\begin{tabular}{|l|l|}
\hline \multicolumn{2}{|c|}{ Ejem plos de términos genéricos extraídos del documento de La Rioja } \\
\hline $\begin{array}{l}\text { Documento que acredite la cobertura } \\
\text { sanitaria de los solicitantes }\end{array}$ & $\begin{array}{l}\text { Término genérico «solicitante», que pierde la } \\
\text { inclusividad al estar acompañado del } \\
\text { masculino «los» }\end{array}$ \\
\hline Fotocopia del DNI de cada solicitante & $\begin{array}{l}\text { Término genérico «solicitante», que mantiene } \\
\text { la inclusividad al estar acompañado del } \\
\text { término genérico «cada» }\end{array}$ \\
\hline $\begin{array}{l}\text { Sí aceptan menores con características, } \\
\text { circunstancias y necesidades especiales }\end{array}$ & $\begin{array}{l}\text { Término genérico «menores», más inclusivo } \\
\text { que «hijos» }\end{array}$ \\
\hline
\end{tabular}

Tabla 4: Términos genéricos de La Rioja

Fuente: Gobierno de La Rioja, Políticas Sociales, Familia, Igualdad y Justicia (2016)

Una vez más, se nos presenta una técnica discursiva efectiva para eliminar las marcas de género, que al igual que los dobletes, debe usarse cuidadosamente para no caer en el uso de artículos masculinos.

\subsection{Fórmulas administrativas}

Hikma 20 (1) (2021), 157 - 184 
En esta ocasión se recalca un elemento que tiende a ser pasado por alto cuando se analizan las posibles estrategias de lenguaje inclusivo, y son las fórmulas tan características, naturales y presentes en este tipo de documentos: los verbos en primera persona y el trato de usted. En más de una ocasión vemos cómo el documento apela y cuestiona directamente a quien lo está rellenando, por lo que se encuentran frases como «¿desea recibir notificaciones?» 0 «deseo que se me notifique por correo electrónico», una forma sencilla y apropiada si se prefiere no usar dobletes, no se encuentra un término genérico inclusivo o si se descarta el uso de caracteres carentes de género (que se expondrá con más detalle a continuación). Esta técnica no resulta artificiosa en documentos burocráticos, y se esquiva la carga de género de, por ejemplo, «el solicitante desea...» si es sustituido por «en caso de que desee...», o simplemente «deseo...». Aun así, se debe recordar que esta técnica también puede generar faltas de concordancia si no se usa de forma constante, ya que el uso de los verbos en primera persona elimina la marca de género, pero ignora la variación de número, otro aspecto a tener en cuenta.

\begin{tabular}{|l|l|}
\hline \multicolumn{2}{|c|}{ Ejem plos de fórmulas administrativas extraídas del documento de Murcia } \\
\hline $\begin{array}{l}\text { ¿Han presentado otra solicitud de } \\
\text { adopción en la Región de Murcia? SI NO }\end{array}$ & $\begin{array}{l}\text { Pregunta directa que elimina por completo la } \\
\text { marca de género }\end{array}$ \\
\hline $\begin{array}{l}\text { EXPONEN: Que todos los datos } \\
\text { consignados en este formulario son } \\
\text { ciertos, lo cual se acredita con la } \\
\text { documentación pertinente }\end{array}$ & $\begin{array}{l}\text { Fórmula directa que elimina por completo la } \\
\text { marca de género }\end{array}$ \\
\hline $\begin{array}{l}\text { SI NO Autorizamos al Órgano } \\
\text { administrativo competente a obtener } \\
{[\ldots . . . j \text { los datos personales }}\end{array}$ & $\begin{array}{l}\text { Respuesta directa que elimina por completo } \\
\text { la marca de género }\end{array}$ \\
\hline
\end{tabular}

Tabla 5: Fórmulas administrativas de Murcia

Fuente: Región de Murcia, Consejería de Familia e Igualdad (s.f.)

Ejemplos de fórmulas administrativas extraídas del documento de Aragón

\begin{tabular}{|l|l|}
\hline $\begin{array}{l}\text { En caso de que Uds. recibieran un menor } \\
\text { en adopción, ¿piensan en adoptar otro } \\
\text { más adelante? SI NO }\end{array}$ & $\begin{array}{l}\text { Pregunta formulada de usted que elimina la } \\
\text { marca de género en la parte adoptante (no en } \\
\text { la adoptada ya que se usa «un menor" y } \\
\text { "Otro") }\end{array}$ \\
\hline $\begin{array}{l}\text { ¿Aceptaría adoptar a un menor con } \\
\text { dificultades físicas y/o sensoriales? SI NO }\end{array}$ & $\begin{array}{l}\text { Pregunta formulada de usted que elimina la } \\
\text { marca de género en la parte adoptante (no en } \\
\text { la adoptada ya que se usa «un menor») }\end{array}$ \\
\hline $\begin{array}{l}\text { Nos comprometemos a: [.... Colaborar con } \\
\text { el personal técnico del IAAS }\end{array}$ & $\begin{array}{l}\text { Afirmación formulada en primera persona que } \\
\text { elimina por completo la marca de género }\end{array}$ \\
\hline
\end{tabular}

Tabla 6: Fórmulas administrativas de Aragón Fuente: Gobierno de Aragón (s.f.)

Hikma 20 (1) (2021), 157 - 184 
Estas fórmulas con la que se dirigen a quienes rellenen el documento, normalmente acompañado de casillas con varias opciones, son tan comunes en este contexto de uso que se pueden encontrar ejemplos en todas las solicitudes analizadas. Cabe destacar que, como se ha expuesto en las tablas, es una fórmula efectiva para eliminar marcas de género en la familia adoptante, pero no para la parte adoptada.

\subsection{Caracteres carentes de marca de género}

En la mayoría de los casos que presenta la lengua castellana, el género viene marcado por un sufijo que denote género masculino, en este caso «o», o que denote femenino, «-a». Así, una de las fórmulas más transgresoras para intentar eliminar la carga de género ha sido la sustitución de estas letras por caracteres considerados neutros, como «@», «X» 0 «e». Esta técnica se ha extendido a nivel cotidiano e informal, y desde hace años pueden apreciarse en carteles, mensajes de texto o incluso el uso de la «e» en el discurso oral o en las redes sociales. En esta ocasión, los ejemplos serían «L@s hij@s», «Lxs hijxs»0 «Les hijes». A pesar de su creciente popularidad a nivel cotidiano, no se han abierto hueco en documentos de índole más burocrática (en parte porque funcionan en el plano escrito, pero plantean dificultades de pronunciación), donde las tres primeras opciones cuentan con más peso.

\section{ANÁLISIS DE FÓRMULAS INCLUSIVAS EN INGLÉS}

\subsection{Dobletes}

En inglés esta técnica está mucho menos presente en comparación con el español debido a que la lengua marca el género de forma diferente. Aun así, no es imposible encontrar textos en los que aparece esta forma de lenguaje inclusivo. La mayoría de los documentos presenta algún tipo de doblete, aunque cabe destacar que son más frecuentes para indicar una variación en número que en género:

\begin{tabular}{|l|l|}
\hline \multicolumn{2}{|c|}{ Ejemplos de dobletes extraídos del documento de Nevada } \\
\hline Aunts/Uncles of the child(ren) & $\begin{array}{l}\text { Dobletes de género en } \\
\text { «aunts/uncles» en plural genérico } \\
\text { Doblete de número en «c hild» }\end{array}$ \\
\hline Siblings of the child(ren) & $\begin{array}{l}\text { «Siblings» en plural genérico } \\
\text { Doblete de número en «child» }\end{array}$ \\
\hline
\end{tabular}

Tabla 7: Dobletes de Nevada

Fuente: Nevada Supreme Court (2017) 
Ejemplos de dobletes extraídos del documento de Massachusetts

\begin{tabular}{|l|l|}
\hline $\begin{array}{l}\text { If petitioner is married, his/her spouse } \\
\text { must sign the petition }\end{array}$ & Dobletes de género \\
\hline $\begin{array}{l}\text { They (he/she) request(s) that the } \\
\text { Court, grant them (him/her) leave to } \\
\text { adopt said child }\end{array}$ & $\begin{array}{l}\text { Dobletes de género y número para } \\
\text { familia adoptante } \\
\text { Sin doblete de número en «c hild» }\end{array}$ \\
\hline
\end{tabular}

Tabla 8: Dobletes de Massachusets

Fuente: Trial Court of Massachusetts Juvenile Court Department (2013)

En esta ocasión, y debido a las propias características del idioma, las faltas de continuidad a la hora de usar esta técnica se reducen considerablemente, y tan solo se detectan al analizar las variaciones de número en los documentos más extensos.

\subsection{Términos genéricos}

Afortunadamente, tal y como se ha especificado en el punto anterior, muchos de los términos del inglés carecen de marca de género, por lo que se nos facilita la tarea. Aun así, no se debe pasar por alto la existencia de palabras que sí denotan género, como los sustantivos «father» 0 «mother». Para eliminar esta realidad binaria que concierne a la parte adoptante, siete de los diecinueve documentos analizados se decantan por «petitioner». Otra tendencia común es el término «parent», acompañado de adjetivos como «adoptive» 0 «prospective»; con menor frecuencia se puede encontrar el uso de «person» para crear esta inclusión en la familia adoptante. Dos de los diecinueve documentos no han mostrado intención de contemplar parejas no heterosexuales, y usan «father»y «mother» sin terceras opciones. Este es el caso de Tennessee y de Virginia Occidental, que podría responder a las visiones políticas de sus líderes.

Ejemplos de términos genéricos extraídos del documento de Florida \begin{tabular}{l|l}
\hline Signature of petitioner & Témino genérico «petitioner»
\end{tabular}

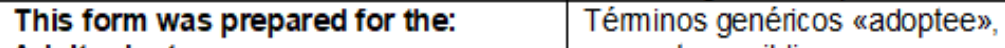
Adult adoptee $\quad$ "parent» $y$ «sibling» Adoptive parent Adult birth sibling \begin{tabular}{|l|l}
\hline This form was completed with the & Término genérico «individual»
\end{tabular} assistance of: (name of individual)

Tabla 9: Términos genéricos de Florida

Fuente: Florida Supreme Court (2015) 


\begin{tabular}{|l|l|}
\hline \multicolumn{2}{|c|}{ Ejem plos de téminos genéricos extraídos del documento de Tennessee } \\
\hline Adoptive father: & $\begin{array}{l}\text { Falta de términos genéricos para la } \\
\text { familia adoptante, uso del masc ulino } \\
\text { Full name of father }\end{array}$ \\
«ather's signature & $\begin{array}{l}\text { Falta de términos genéricos para la } \\
\text { familia adoptante, uso del femenino } \\
\text { Adoptive mother. }\end{array}$ \\
$\begin{array}{l}\text { Full legal name of mother } \\
\text { Maiden sumame of mother } \\
\text { Mother's signature }\end{array}$ & $\begin{array}{l}\text { posibles diferencias entre el nombre } \\
\text { de soltera y de casada. }\end{array}$ \\
\hline
\end{tabular}

Tabla 10: Términos genéricos de Tennessee

Fuente: State of Tennessee, Department of Health (2017)

Otro aspecto llamativo es que en cinco de estos documentos se ofrecen tablas para que las cumplimente la familia adoptante, y se crea una combinación de las dos realidades vistas anteriormente. Es decir, se puede dar el caso de que presenten «father», «mother» y «parent» o «petitioner» en los apartados, ofreciendo todas las combinaciones posibles. El hecho de que no se limite a «father» $y$ «mother» en las dos casillas puede sugerir una inclusión real para todas las personas de género no binario, aunque el único caso evidente y directo de representación no binaria se presenta a continuación, en el apartado «representación de género». También merece ser mencionada la diferencia en cuanto al uso léxico entre la familia adoptante y biológica, que se explica más en detalle a continuación.

\begin{tabular}{|c|c|}
\hline \multicolumn{2}{|c|}{ Ejemplos de términos genéricos extraídos del documento de Texas } \\
\hline $\begin{array}{l}\text { Parent: } \\
\text { Adoptive/biological } \\
\text { Mother } \\
\text { Father } \\
\text { Parent }\end{array}$ & $\begin{array}{l}\text { Dos apartados idénticos para la } \\
\text { familia adoptante que ofrecen tres } \\
\text { opciones léxicas, dos con marca de } \\
\text { género y una neutra }\end{array}$ \\
\hline $\begin{array}{l}\text { Biological mother: } \\
\text { First } \\
\text { Middle } \\
\text { Last (maiden) }\end{array}$ & $\begin{array}{l}\text { Falta de términos genéricos para la } \\
\text { familia biológica, uso del femenino } \\
\text { «mother» y se solicita el apellido de } \\
\text { soltera }\end{array}$ \\
\hline $\begin{array}{l}\text { Biological father. } \\
\text { First } \\
\text { Middle } \\
\text { Last }\end{array}$ & $\begin{array}{l}\text { Falta de términos genéricos para la } \\
\text { familia biológica, uso del masculino } \\
\text { «father» }\end{array}$ \\
\hline
\end{tabular}

Tabla 11: Términos genéricos de Texas

Fuente: State of Texas (2015) 
La presencia de la parte adoptada es esencial en estos documentos, y en dieciocho de los diecinueve documentos, se refieren en algún punto a esta parte con el término neutro «child». La excepción se encuentra en Florida, que se decanta por «adoptee». Otras opciones inclusivas que se encuentran como sinónimo en estos textos incluyen «juvenile», «infant» e incluso «person who is being adopted». Cabe destacar que diez de los documentos en inglés solicitan indicar el género de esta parte, y si tan solo se ofrecen dos casillas binarias para marcarlo, se elimina de un plumazo toda representación no binaria que podría conceder la terminología neutra. En cambio, si se facilita una casilla para rellenar con texto libre, al igual que con el resto de los apartados, no se pierde la inclusión que se viene demandando.

\begin{tabular}{|l|l|}
\hline \multicolumn{2}{|c|}{ Ejemplos de téminos genéricos extraídos del documento de Arizona } \\
\hline The clerk of the court must complete & Término genérico «child» \\
Part III and confirm [...] that the & \\
child's new adoptive name to appear & \\
on the birth certificate if accurate. & \\
\hline $\begin{array}{l}\text { The adoptee or the adoptive parent } \\
\text { must complete Part IV. In the event } \\
\text { there is an error [...] will contact the }\end{array}$ & Término genérico «adoptee» \\
Adoptee & \\
\hline
\end{tabular}

Tabla 12: Términos genéricos de Arizona

Fuente: Arizona Department of Health Service. (s.f.)

Finalmente, es preciso mencionar que la inclusión se ve claramente reducida al hablar de la familia biológica, mencionada en once documentos. En diez de estos archivos se habla de forma binaria para referirse a la familia que da en adopción; la única excepción que rechaza la norma es el caso de Nueva Jersey, que ofrece «Biological: Mother/Parent A» y «Biological: Father/Parent B». Estas opciones favorecen un modelo heterosexual y tradicional de la familia, pero no lo reducen a la única opción viable. Uno de los ejemplos claros se trata en la tabla 11 y se repite en otros documentos analizados. 


\begin{tabular}{|l|l|}
\hline \multicolumn{2}{|c|}{ Ejem plos de términos genéricos extraídos del documento de New Mexico } \\
\hline Natural mother information & $\begin{array}{l}\text { Falta de términos genéricos para la } \\
\text { familia biológica, uso del femenino } \\
\text { «mother» }\end{array}$ \\
\hline Natural father information & $\begin{array}{l}\text { Falta de términos genéricos para la } \\
\text { familia biológica, uso del masculino } \\
\text { «father» }\end{array}$ \\
\hline Father's information or parent 1 & $\begin{array}{l}\text { Témino masculino y término neutro } \\
\text { para la familia adoptante }\end{array}$ \\
\hline $\begin{array}{l}\text { Mother's information (Maiden name- } \\
\mathbf{2}\end{array}$ & $\begin{array}{l}\text { Término femenino y término neutro } \\
\text { para la familia adoptante }\end{array}$ \\
\hline
\end{tabular}

Tabla 13: Términos genéricos de New Mexico

Fuente: Second Judicial District Court of New Mexico (s.f.)

\subsection{Fórmulas administrativas}

En el caso de los documentos norteamericanos, el trato de usted queda descartado porque la lengua no marca diferencia en la conjugación, pero las preguntas y respuestas en primera persona son tan características en estos documentos como en España. En inglés destacan expresiones como «l hereby certify [...]» 0 «l, (name)__, consent to [...]». También podemos encontrar, al igual que en español, preguntas acompañadas de casillas que indican «yes» 0 «no».

\begin{tabular}{|l|l|}
\hline \multicolumn{2}{|c|}{$\begin{array}{l}\text { Ejemplos de fómmulas administrativas extraídas del documento de } \\
\text { Delaware }\end{array}$} \\
\hline $\begin{array}{l}\text { I (do/do not) wish to request a } \\
\text { hearing/ceremony if the adoption is } \\
\text { granted }\end{array}$ & $\begin{array}{l}\text { Respuesta directa que elimina por } \\
\text { completo la marca de género }\end{array}$ \\
\hline $\begin{array}{l}\mathrm{I} \text {, (name) state the information } \\
\text { in this Petition for Adoption is true }\end{array}$ & $\begin{array}{l}\text { Fómula directa que elimina por } \\
\text { completo la marca de género }\end{array}$ \\
\hline
\end{tabular}

Tabla 14: Fórmulas administrativas de Delaware

Fuente: Family Court of the State of Delaware (2011)

En esta ocasión, la frecuencia de uso de esta técnica es elevada pero no total, por lo que es menos popular que en español en cuanto a los documentos analizados en este estudio. Recordamos que estas fórmulas en primera persona son efectivas para eliminar la marca de género en numerosas ocasiones, pero pueden causar incongruencias de número. 


\subsection{Uso del neutro «they»}

$\mathrm{Al}$ igual que los caracteres carentes de género españoles, el uso del pronombre neutro «they» está ganando peso y popularidad en el discurso oral y cotidiano (en parte porque es un pronombre propio de la lengua, solo estaríamos hablando de un neologismo de significado). Actualmente es posible encontrar varios ejemplos en los que se usa "they/them» cuando prefiere no especificarse el género. Además, algunas personas angloparlantes no binarias solicitan a su entorno que lo usen en las frases donde se les mencione. Esta herramienta sobre el uso del «they/them», y de los plurales en general, puede ser muy útil cuando se usen frases o normas genéricas. De la misma forma que los caracteres carentes de género, el uso del «they» no está presente en ninguno de estos documentos, que se decantan el uso de las primeras técnicas para crear inclusión.

\section{REPRESENTACIÓN DE GÉNERO}

En este apartado se analiza la frecuencia con la que se solicita específicamente indicar el género de alguna de las personas involucradas en el proceso de adopción. Se han analizado seis documentos en español, y de estos seis, uno solicitaba indicaciones binarias bajo el término "género» y otro bajo "sexo". Esto nos deja un total de cuatro documentos que no lo solicitan de forma directa. En inglés, de un total de diecinueve, cuatro solicitan «gender» y seis «sex». De estos diez documentos totales que solicitan «gender» O «sex», la mitad ofrecen casillas binarias para determinar «male» o «female» y la otra mitad incluyen casillas en las que se puede escribir lo que se desee. El caso especial es el de Nueva Jersey, que ofrece bajo el término «sex» una tercera casilla «Undesignated/Non-Binary»

\begin{tabular}{|c|c|}
\hline \multicolumn{2}{|c|}{$\begin{array}{l}\text { Ejemplo de representación de género extraído del documento de New } \\
\text { Jersey }\end{array}$} \\
\hline $\begin{array}{l}\text { Sex: } \\
\text { Male } \\
\text { Female } \\
\text { Undesignated/non-binary }\end{array}$ & $\begin{array}{l}\text { Muestra directa de rec onocimiento } \\
\text { no binario referido a la parte } \\
\text { adoptada }\end{array}$ \\
\hline
\end{tabular}

Tabla 15: Fórmulas administrativas de New Jersey

Fuente: State of New Jersey (2019)

Por todo esto, cabe destacar que la tendencia más general tanto en inglés como en español es la obligación de solicitar indicaciones de género en los documentos de forma binaria. Por desgracia, los apartados que ofrecen tan solo dos opciones tienen una presencia importante, eliminando de raíz toda representación fluida, aunque los apartados que permiten rellenar con 
texto este apartado y la tercera casilla de Nueva Jersey nos abren un abanico de opciones mucho más amplio y representativo.

Asimismo, resulta llamativo el intercambio de términos "sexo» y "género». Últimamente, y según las definiciones de la RAE y el MerriamWebster, el sexo parece referirse a características biológicas, mientras que el género engloba actitudes, comportamientos y modelos que pertenecen a la esfera de lo social. Sin embargo, Grijelmo (2019) señala el uso indebido del término «género» cuando realmente estamos hablando de «sexo» (2019, p. 151-159): sucede, por ejemplo, en la expresión «violencia de género», que puede entenderse de forma eufemística cuando realmente se habla de «violencia machista», que no afecta a los dos sexos (pero el género sí engloba ambos):

Frente a esa univocidad indudable de machista, el término género altera su polaridad según el contexto: en violencia de género, esta voz, al sustituir a machista, refleja una idea firmemente peyorativa. Sin embargo, la locución políticas de género puede equivaler a políticas de igualdad, y de tal modo ese género adquiere un tinte positivo, como sucede también en conciencia de género y otras construcciones similares (Grijelmo, 2019, p. 159; cursiva en el original).

Igualmente, conviene retomar el hecho de que se tienda a una mayor inclusión en lo que respecta a la familia adoptante, haciendo hincapié en un modelo heteropatriarcal y binario en lo que concierne a la parte biológica, y no digamos a la parte adoptada. La aceptación social de configuraciones trans en menores es un debate candente en España, especialmente en 2017, cuando el autobús de la organización Hazte Oír recorrió las calles con el mensaje «Los niños tienen pene. Las niñas tienen vulva. Que no te engañen. Si naces hombre, eres hombre. Si eres mujer, seguirás siéndolo». Ahora, la Proposición de Ley 122/000191 mencionada en la introducción, también es objeto de debate, condenada por algunas voces dentro y fuera del feminismo que argumentan que diluirá el concepto de «mujer» y suscitará más desigualdades.

\section{RESULTADOS}

En aras de la claridad, se recogen en este epígrafe por separado los resultados obtenidos en el apartado de análisis. Siguiendo dicha estructura, se ofrecerán las tendencias en español, seguidas del inglés y, por último, la obligación de consignar el género en estos documentos. 


\subsection{Resultados del análisis de la muestra en español}

La primera estrategia analizada es el uso de dobletes. En cuatro de seis documentos se observaron dobletes y en todos aparecían faltas de continuidad, lo que no solo refleja inconsistencia en el uso, sino que además lleva a incongruencias gramaticales en algunos casos. En lo que respecta al uso de sustantivos genéricos, si bien es cierto que en todos los documentos se han observado intentos de uso de este mecanismo, se ha detectado que las características del español plantean un problema parecido al de los dobletes, pues al concordar en plural se suele eliminar la inclusión. En este punto, además, cabe destacar que el trato hacia la parte adoptada suele ser más inestable (y se hace referencia a ella con la denominación «hijos», en lugar de «menores»). También se observaron fórmulas administrativas que podrían considerarse inclusivas, como son los casos de Murcia y Aragón.

Ninguno de los documentos analizados incorporaba fórmulas alternativas, como la @ o la -x.

\subsection{Resultados del análisis de la muestra en inglés}

El inglés plantea una menor necesidad de uso de dobletes, por lo que solo se sugieren dos ejemplos de diecinueve documentos analizados. En este sentido, la falta de continuidad se hace más difícil de detectar debido a su presencia reducida. Se ha observado el uso de sustantivos genéricos en siete documentos, que optan por sustantivos como «petitioner» en lugar de otros que marcan el género, como «father» 0 «mother». Es relevante destacar que en cinco documentos se ofrece una tabla para rellenar con texto libre, lo que conlleva más posibilidades de inclusión.

Al igual que sucede con el español, el tratamiento hacia la parte adoptada difiere: en dieciocho documentos se refieren a ella como "child», tan solo en uno se opta por "adoptee» y en diez casos se establece la necesidad de incluir el género. De la misma manera, en lo que respecta a la familia biológica, disminuye el nivel de inclusión: en once solicitudes se observan categorías binarias (con la notable excepción de Nueva Jersey).

El empleo de fórmulas administrativas es menor que en España, aunque sí se observan ejemplos, como el de Delaware. En ningún caso se han observado otros mecanismos, como el uso de «they» para ciertas identidades.

\subsection{Obligación de consignar el género}

En el caso de España, una de las solicitudes obligaba a marcarlo con el sustantivo "género», y otra con la denominación "sexo». Los cuatro documentos restantes no requerían hacerlo de forma directa. 
En el caso de Estados Unidos, cuatro de los diecinueve lo especificaban con el inglés "gender», y otros seis con el sustantivo «sex». En cinco de estos documentos se pedía hacerlo mediante casillas, pero lo relevante es que en otros cinco se permitía rellenar una celda con texto libre, lo que ofrece más opciones de inclusión. La excepción, de nuevo, es la de Nueva Jersey, con una casilla que ofrecía la opción de «Undesignated/Non binary».

\section{CONCLUSIONES}

Planteamos como objetivo en la introducción de este artículo un estudio acerca de las diferentes estrategias que ofrecen el español y el inglés para lograr una mayor inclusión a través del lenguaje, pues nuestra hipótesis consideraba que la representación que se daba de ciertos sectores del colectivo LGTBIQ+ era insuficiente. Después de observar los resultados del análisis, consideramos que se puede confirmar este planteamiento, pues, aunque indudablemente se han hecho intentos de avanzar, aún queda trayecto por recorrer en lo que respecta especialmente a la representación de personas trans o no binarias. Al estudiar una muestra de documentos que pertenecen a esa "lengua cultivada» de la que hablábamos en la parte teórica, entendemos que ofrecen una posibilidad de reflexión y selección previa de las fórmulas que se van a utilizar, con un proceso de revisión. En situaciones informales, los hablantes presentan otros usos de la lengua, y la espontaneidad del lenguaje oral hace casi imposible la incorporación de las estrategias que aquí se defienden.

Tanto el inglés como el español recurren a los manidos dobletes que, como se ha observado, presentan una dificultad de consistencia y aumentan sensiblemente la extensión del texto, un aspecto que debe tenerse en cuenta en la elaboración de formularios y casillas. Además, no resultan del todo efectivos, pues en algunas ocasiones, aunque se añadan varios de ellos en la misma oración, no terminan de recoger todas las posibilidades que, quizás, sí consideraba el organismo que emite el documento. Los genéricos, otra de las opciones que contempla Grijelmo (2019) y que pueden ser muy funcionales, pierden toda su esencia cuando se utilizan a la ligera o se acompañan de dobletes que determinan la carga semántica. Desde aquí se defiende la posibilidad de utilizar las fórmulas asentadas del lenguaje jurídicoadministrativo como formas de lenguaje inclusivo: nos referimos a declaraciones en primera persona o a preguntas directas que eliminan la carga de género. Están presentes y aceptadas socialmente en la lengua, no plantean problemas de registro o de extensión y no suponen dificultades para ser oralizadas. 
Por último, en lo que respecta al uso de neologismos o de morfemas novedosos, como el pronombre «elle» o el sufijo «-e», coincidimos con Maldonado (2017) en que abren posibilidades interesantes, al igual que el uso de «they» en inglés, sobre todo para el colectivo no binario. Sin embargo, como señala Grijelmo (2019), son fórmulas que pueden tardar en encontrar acomodo en el lenguaje oral (no surgen de forma espontánea para la mayoría) o en el plano administrativo.

Aunque no tenemos certeza de cómo evolucionarán estas tendencias, defendemos la necesidad de que se utilice el lenguaje inclusivo, pues en ciertos contextos, lo que para algunos públicos puede constituir un gesto simbólico, para otras personas marca la diferencia: reconocer la identidad propia validada por las instituciones acaba con la violencia simbólica $y$, potencialmente, con la explícita que puede sufrir el colectivo. La representación legal y política es una cuestión ética y un derecho.

REFERENCIAS BIBLIOGRÁFICAS

Alexander, J. (2003). Bisexuality and Transgenderism: InterSEXtions of the Others. New York: Haworth Press.

Arizona Department of Health Services. (s.f.). Certificate of adoption instruction guide. Obtenido de https://azdhs.gov/documents/vitalrecords/manuals/certificate-of-adoption-adult.pdf

Arkansas Department of Human Services. (s.f.). Adoption Information Sheet. Obtenido de https://humanservices.arkansas.gov/

Baker, M. (2006). Translation and Conflict: A Narrative Account. London: Routledge.

Beard, M. (2017). Women \& Power: A Manifesto. New York: Liveright.

Beauvoir, S. (1949/2016). El segundo sexo. Barcelona: Publilibre.

Braidotti, R. (2006/2009). Transposiciones. Sobre la ética nómada. Barcelona: Gedisa.

Butler, J. (1990/1999). Gender Trouble: Feminism and the Subversion of Identity. New York: Routledge.

Butler, J. (1995/2002). Cuerpos que importan: sobre los límites materiales y discursivos del "sexo". México D. F.: Paidós.

Colorado Judicial Branch. (s.f.). Report of Adoption. Obtenido de https://www.courts.state.co.us/Forms/SubCategory.cfm?Category=Ad option 
Comunidad de Madrid, Asuntos Sociales. (2015). Adopción. Obtenido de https://www.comunidad.madrid/servicios/asuntos-sociales

Consell de Mallorca, Instituto Mallorquín de Asuntos Sociales. (s.f.). Valoración de la idoneidad para la adopción nacional. Obtenido de https://seu.conselldemallorca.net/es/web/seu/ficha?key=12213

Cronin, M. (2006). Translation and Identity. London: Routledge.

Department of Child Services of Indiana. (s.f.). Indiana Adoption Program Application. Obtenido de https://www.in.gov/dcs/2831.htm

Derrida, J. (1977/2001). On Cosmopolitanism and Forgiveness. London: Routledge.

Derrida, J. (2000). Hostipitality. Angelaki, Journal of the Theoretical Humanities, 5(3), 3-18.

Family Court of the State of Delaware. (2011). Peition for Adoption. Obtenido de https://courts.delaware.gov/family/adoption/forms.aspx

Florida Supreme Court. (2015). Petition for Adoption. Obtenido de https://www.florida-court-forms.net/florida-adoption-forms.html

Foucault, M. (1975/2002). Vigilar y castigar: el nacimiento de la prisión. Buenos Aires: Siglo Veintiuno Editores.

Foucault, M. (1976/2007). Historia de la sexualidad: la voluntad del saber. México D. F.: Siglo Veintiuno Editores.

Franco, A. (2019). ¿Todos, todos/as, todxs o todes? Efectos cognitivos del uso del genérico masculino y sus formas alternativas en español. Barcelona: Egales.

Generalitat de Catalunya. (2020). Petición genérica sin firma electrónica. Obtenido de https://web.gencat.cat/es/

Gobierno de Aragón. (s.f.). Cuestionario Adopción Nacional (Valoración de Idoneidad). Obtenido de https://www.aragon.es/-/adopcion-nacional

Gobierno de La Rioja, Políticas Sociales, Familia, Igualdad y Justicia. (2016). Solicitud del procedimiento de valoración e dioneidad para la adopción nacional. Obtenido de https://www.larioja.org/serviciossociales/es/infancia/adopcion

Grijelmo, A. (2019). Propuesta de acuerdo sobre el lenguaje inclusivo. Madrid: Taurus. 
Horno, M. (2018). Bondades, peligros y redundancias del lenguaje inclusivo. Letras libres.

House, J. M. (2005). Introduction. En J. M. House, Translation and the Construction of Identity. IATIS Yearbook 2005 (págs. 3-13). Cornwall: T. J. International.

Judicial Branch of the State of New Hampsire. (2009). Petition for Adoption. Obtenido de https://www.courts.state.nh.us/forms/nhjb-2185-fp.pdf

Judicial Council of California. (2018). Adopt-200 Adoption Request. Obtenido de https://www.courts.ca.gov/forms.htm?query=adoption

Maldonado, L. (18 de Junio de 2017). La lengua no tiene sexo: «Elle está cansade». El Español.

Manzanas, A. B. (2017). Hospitality in American Literature and Culture. Spaces, Bodies, Borders. London: Routledge.

Martínez Pleguezuelos, A. (2018). Traducción e identidad sexual: reescrituras audiovisuales desde la Teoría Queer. Granada: Comares.

Michigan Department of Health and Human Services. (2010). Adoption Closing Summary. Obtenido de https://www.michigan.gov/mdhhs/0,5885,7-339-71551_7199_52659--,00.html

Miller, C. S. (1995). The Handbook of Non-Sexist Writing for Writers, Editors and Speakers. London: The Women's Press.

Nevada Supreme Court. (2017). Petition For Adoption. Obtenido de http://selfhelp.nvcourts.gov/self-help/adoption-termination-of-parentalrights/how-to-file-for-adoption/filing-the-adoption-case

New York State, Office of children and family services. (2011). Application to adopt. Obtenido de https://ocfs.ny.gov/main/Forms/adoption/LDSS0857\%20Application\%20to\%20Adopt\%20\%20Part\%201\%20Family\%20Adoption\%20Registry\%20Information.p df

Real Academia Española: Diccionario de la lengua española. (s.f.). 23. ${ }^{a}$ ed., [versión 23.3 en línea].

Región de Murcia, Consejería de Familia e Igualdad. (s.f.). Solicitud de declaración de idoneidad para la adopción nacional. Obtenido de https://www.carm.es/web/pagina?IDCONTENIDO=6046\&IDTIPO=100 \&RASTRO $=c 560 \$ \mathrm{~m}$ 
Ricoeur, P. (2005). Sobre la traducción. Barcelona: Paidós.

Sánchez, M. y Fernández, M. (2019). MariCorners: investigaciones queer en la academia. Barcelona: Egales.

Second Judicial District Court of New Mexico. (s.f.). Report of Adoption. Obtenido de https://seconddistrictcourt.nmcourts.gov/childrens-courtself-help-forms.aspx

State of New Jersey. (2019). Report of Adoption. Obtenido de https://www.njcourts.gov/selfhelp/catalog.html?keywords=Adoption\%2 Oset\&customer=Prose

State of Tennessee, Department of Health. (2017). Certificate of Adoption. Obtenido

de https://www.tn.gov/content/dam/tn/health/documents/vital-records/PH1248-Certificate-of-Adoption.pdf

State of Texas. (2015). Certificate of Adoption. Obtenido de http://www.dshs.texas.gov

State of West Virginia. (2011). Certificate of Adoption. Obtenido de http://www.wvdhhr.org/bph/hsc/vital/forms/certificate_of_adoption_notf illable_07_06_2011.pdf

Superior Court for the State of Alaska. (s.f.). Petition for Adoption. Obtenido de https://public.courts.alaska.gov/web/forms/docs/p-405.pdf

Trial Court of Massachusetts Juvenile Court Department. (2013). Petition for the adoption of a minor. Obtenido de https://www.mass.gov/how-to/filefor-adoption

Utah Department of Health. (2015). Report of Adoption. Obtenido de https://vitalrecords.utah.gov/wp-content/uploads/Report-ofAdoption.pdf

Vidal, A. (2014). Translating Hybrid Literatures From Hostipitality to Hospitality. Translation and Ethnicity, European Journal of English Studies (18)3, 242-262. 